\title{
Editorial
}

\section{Emerging Machine Learning Techniques in Signal Processing}

\author{
Theodoros Evgeniou, ${ }^{1}$ Aníbal R. Figueiras-Vidal, ${ }^{2}$ and Sergios Theodoridis ${ }^{3}$ \\ ${ }^{1}$ Technology Management and Decision Sciences, INSEAD, Boulevard de Constance, 77300 Fontainebleau, France \\ ${ }^{2}$ Department of Signal Theory and Communications, Carlos III University of Madrid, 28911 Leganés, Madrid, Spain \\ ${ }^{3}$ Deptartment of Informatics and Telecommunications, Division of Communications and Signal Processing, \\ Panepistimiopolis, Ilissia, Athens 15784, Greece
}

Correspondence should be addressed to Sergios Theodoridis, stheodor@di.uoa.gr

Received 28 July 2008; Accepted 28 July 2008

Copyright () 2008 Theodoros Evgeniou et al. This is an open access article distributed under the Creative Commons Attribution License, which permits unrestricted use, distribution, and reproduction in any medium, provided the original work is properly cited.

In the era of knowledge-based society and machine automation, there is a strong interest in machine learning (ML) techniques in a wide range of applications. The attention paid to ML methods within the DSP community is not new. Speech recognition is an example of an area where DSP and machine learning have been combined to develop efficient and robust speech recognizers. Channel equalization is another area at the intersection of ML and DSP techniques. After all, deciding upon the transmitted information symbol is nothing but a class assignment task. In cognitive radio, DSP techniques and ML methods can work together for developing algorithms for the efficient utilization of the radio spectrum. Image/video/audio coding, recognition, and retrieval are some additional typical examples where DSP and ML tie together. Another problem at the heart of the DSP community interests is the regression task, which can be cast as an ML problem. Biomedical applications constitute another area in which mixed ML and DSP ideas proved to be useful.

Over the past years, a number of new powerful ML techniques have been developed, which are suitable for nonlinear processing and the general case of non-Gaussian data, and also for nondifferentiable cost functions or cost functions referring to robust statistics. Adaptive versions of some of these techniques have only recently started being studied. This is an area in which the DSP community has a lot to say and contribute.

The focus of this special issue is twofold: (a) to consider novel theoretical results in ML methods and algorithms in the light of typical DSP applications, and (b) to report novel results obtained by the application of ML techniques in some typical DSP tasks.
The special issue consists of 14 accepted papers. The papers can be divided into two main categories: (a) theory and algorithms and (b) applications. The latter covers a rather broad range of areas, such as communications, audio processing and recognition, medicine and neuroscience, and implementation architectures.

The paper authored by H. Li and T. Adali extends previous works on using complex-valued calculus in order to implement nonlinear adaptive signal processing algorithms in the complex domain, as well as some procedures resulting from this framework.

The paper authored by W. Liu and J. C. Príncipe presents a general framework for using the "kernel trick" in the context of affine projection algorithms, as well as some procedures resulting from this framework.

The paper authored by K. Slavakis and S. Theodoridis deals with the development of a novel adaptive algorithm for nonlinear classification/system identification, using kernels. The algorithm is derived in the general framework of convex optimization.

The paper authored by S. V. Vaerenbergh et al. treats the identification of nonlinear systems. A supervised identification approach is employed, and it is shown how the kernel canonical correlation analysis (KCCA) emerges as the logical solution to this problem.

The paper authored by J. L. Rojo-Álvarez et al. presents a novel algorithm for sparse deconvolution, which is developed within the SVM framework. The performance of the algorithm is analyzed with respect to its robustness in the presence of non-Gaussian additive noise.

The paper authored by Guang Deng proposes a family of sequential algorithms based on the Bayesian formulation of 
the problem to solve the M-estimate of a linear observation model. The maximum a posteriori (MAP) estimation leads to RLS- and LMS-type algorithms.

The paper authored by D. J. Miller et al. presents a transductive extension of maximum entropy/improved iterative scaling for aggregation in distributed classification.

The paper authored by K. M. Guan et al. studies the optimization of the performance of a level-crossing analogue-todigital converter (LC ADC) by providing several sequential algorithms that adaptively update the ADC reference levels.

The paper authored by F. Pérez-Cruz and J. J. MurilloFuentes describes how to use Gaussian processes-based regression and decision designs to build nonlinear receivers for digital communications, comparing their performances with those of previously proposed methods.

The paper authored by A. Rabaoui et al. applies optimized one-class support vector machines (1-SVMs) to tackle sound detection as well as classification tasks in the context of a sound recognition process.

The paper authored by W. Wang et al. presents a novel approach for onset detection of musical notes from audio signals. New detection functions are constructed from linear temporal bases that are obtained from the decomposition of musical spectra using nonnegative matrix factorization (NMF).

The paper authored by G. Wang et al. shows how AR models, which are based on minimizing a new cost function, mean-square cross-prediction error, are useful to extract the atrial activity signal from ECG registers.

The paper authored by C. Zhang et al. deals with the task of estimating the visual display terminal (VDT) mental fatigue. The kernel principal component analysis (KPCA) and hidden Markov model (HMM) are combined to differentiate two mental fatigue states and effectively reduce the dimensions of the required feature vectors.

The final paper authored by S. Darmanjian and J. C. Príncipe introduces two new algorithms, that is, boosted mixtures and linked mixtures of hidden Markov chains, to decompose joint likelihoods into marginal likelihoods, demonstrating their simplicity and potential to be applied in brain machine interfaces.

We expect that this special issue will be well received by the DSP and ML communities, and that it will be a step forward in strengthening research in the intersection of these two significant disciplines.

Theodoros Evgeniou Aníbal R. Figueiras-Vidal

Sergios Theodoridis 